\title{
Effect of pore water velocities and solute input methods on chloride transport in the undisturbed soil columns of Loess Plateau
}

\author{
BeiBei Zhou' ${ }^{1}$ QuanJiu Wang'
}

Received: 2 April 2015/Accepted: 31 March 2016/Published online: 9 April 2016

(c) The Author(s) 2016. This article is published with open access at Springerlink.com

\begin{abstract}
Studies on solute transport under different pore water velocity and solute input methods in undisturbed soil could play instructive roles for crop production. Based on the experiments in the laboratory, the effect of solute input methods with small pulse input and large pulse input, as well as four pore water velocities, on chloride transport in the undisturbed soil columns obtained from the Loess Plateau under controlled condition was studied. Chloride breakthrough curves (BTCs) were generated using the miscible displacement method under water-saturated, steady flow conditions. Using the $0.15 \mathrm{~mol} \mathrm{~L}^{-1} \mathrm{CaCl}_{2}$ solution as a tracer, a small pulse ( 0.1 pore volumes) was first induced, and then, after all the solution was wash off, a large pulse ( 0.5 pore volumes) was conducted. The convection-dispersion equation (CDE) and the two-region model (T-R) were used to describe the BTCs, and their prediction accuracies and fitted parameters were compared as well. All the BTCs obtained for the different input methods and the four pore water velocities were all smooth. However, the shapes of the BTCs varied greatly; small pulse inputs resulted in more rapid attainment of peak values that appeared earlier with increases in pore water velocity, whereas large pulse inputs resulted in an opposite trend. Both models could fit the experimental data well, but the prediction accuracy of the T-R was better. The values of the dispersivity, $\lambda$, calculated from the dispersion coefficient obtained from the CDE were about one order of magnitude larger than those calculated from the dispersion
\end{abstract}

BeiBei Zhou

happyangle222@aliyun.com

1 State Key Laboratory of Eco-Hydraulic Engineering, Institute of Water Resources and Hydro-electric Engineering, Xi' an University of Technology, Xi' an 710048, China coefficient given by the T-R, but the calculated Peclet number, $P e$, was lower. The mobile-immobile partition coefficient, $\beta$, decreased, while the mass exchange coefficient increased with increases in pore water velocity.

Keywords Solute transport - CDE - Two-region model · Pore water velocity
Abbreviations
BTCs Breakthrough curves
CDE Convection-dispersion equation
T-R Two-region model
SSQ Sum of squares residual value
Exp Experimental

\section{Introduction}

Soil water hydrological process controls the transport of pollutants, such as pesticides from agricultural land, either in overland flow or in percolating water that may enter the groundwater. Concerns about these have motived numerous experiments and theoretical studies relating soil properties to soil water movement and chemical transport (Zhou 2009; Beven and Germanm 1982). However, due to the soil genesis and human activity, it always has a heterogeneous hydraulic characteristic in the soil profile (Anderson and Bouma 1977). As a result, water flow through the soil profile can allow surface-applied agricultural chemicals to move through the root zone at different rate and quantities, posing problems of economic loss and deteriorating the wicked environment.

Laboratory studies conducted with columns of soil material have been used to examine the dependence of 
water and solute transport on soil structure (Kanchanasut et al. 1978), soil texture (Coats and Smith 1964), soil water content (Elrick and French 1966), initial soil water content (White 1985; White et al. 1986) on water and solute application (Seyfried and Rao 1987). But most of them were based on the disturbed soil column with a small size. Besides, less research has been undertaken to examine the effect of the solute application methods and of the pore water velocities on solute transport in large undisturbed soil columns. This question is pertinent to the formulation and validation of transport models for water flow and is relevant to water and chemical management. And the detection of solute transport in soils is commonly achieved by applying a chemical tracer to the soil surface and measuring its concentration over time at a certain depth to produce a breakthrough curve (BTC) (Haws and Das 2004).

An understanding of the effect of pore water velocity and input method on solute transport processes is needed in order to predict the movement of agricultural chemicals through the soils of this region, which is helpful with the improvement techniques for managing the agricultural application. The objectives of this study were as follows: (1) to examine the effect of solute input methods and pore water velocity on solute transport in loessial soil columns and (2) to evaluate the convection-dispersion equation (CDE) and the two-region model (T-R) for predicting nonreactive solute BTCs in large undisturbed soil column of the Loess Plateau.

\section{Materials and methods}

\section{Model background and development}

The earliest models based on chemical and physical nonequilibrium were the CDE and the T-R (Skopp and Warrick 1974; van Genuchten and Wierenga 1976).

The most commonly used model CDE (Lapidus and Amundson 1952) is characterized by a convection term with mean pore water velocity $V\left(\mathrm{~cm} \mathrm{~h}^{-1}\right)$ and a hydrodynamic dispersion coefficient $D\left(\mathrm{~cm}^{2} \mathrm{~h}^{-1}\right)$ :

$R \frac{\partial C}{\partial t}=D \frac{\partial^{2} C}{\partial x^{2}}-V \frac{\partial C}{\partial x}$

where $C$ is the solute concentration $\left(\mu \mathrm{g} \mathrm{mL}^{-1}\right), t$ is the time (h), $x$ is the spatial coordinate $(\mathrm{cm})$, and $R$ is the retardation factor. The following boundary and initial conditions are commonly applied for the CDE:

$C=0 \quad t=0, \quad 0<x<L$

$V C_{0}=-D(\partial C / \partial x)+V C \quad x=0, \quad 0<t<t_{\mathrm{p}}$
$0=-D(\partial C / \partial x)+V C \quad x=0, \quad t>t_{\mathrm{p}}$

$\partial C / \partial x=0 \quad x=L, \quad t>0$

where $L$ is the solute transport length $(\mathrm{cm}), t_{\mathrm{p}}$ is the duration of a solute pulse input (h), and $C_{0}$ is the solute concentration in the applied pulse $\left(\mu \mathrm{g} \mathrm{mL}^{-1}\right)$.

The T-R, derived by van Genuchten and Wagenet (1989), is given by:

$\theta_{\mathrm{m}} \frac{\partial C_{\mathrm{m}}}{\partial t}+\theta_{\mathrm{im}} \frac{\partial C_{\mathrm{im}}}{\partial t}=\theta_{\mathrm{m}} D \frac{\partial^{2} C_{\mathrm{m}}}{\partial x^{2}}-V_{\mathrm{m}} \theta_{\mathrm{m}} \frac{\partial C_{\mathrm{m}}}{\partial x}$

$\theta_{\mathrm{im}} \frac{\partial C_{\mathrm{im}}}{\partial t}=\omega\left(C_{\mathrm{m}}-C_{\mathrm{im}}\right)$

$\theta=\theta_{\mathrm{m}}+\theta_{\mathrm{im}}$

where $\theta$ is the volumetric water content $\left(\mathrm{cm}^{3} \mathrm{~cm}^{-3}\right) ; \theta_{\mathrm{m}}$ and $\theta_{\mathrm{im}}$ are the volumetric water contents in the immobile and mobile regions, respectively; $C_{\mathrm{m}}$ and $C_{\mathrm{im}}$ are the concentrations in the immobile and mobile regions $\left(\mu \mathrm{g} \mathrm{mL}^{-1}\right) ; D$ is the diffusion coefficient $\left(\mathrm{cm}^{2} \mathrm{~h}^{-1}\right) ; V_{\mathrm{m}}$ is the mean pore water velocity in the mobile region $\left(\mathrm{cm} \mathrm{h}^{-1}\right) ; t$ is the flow time $(\mathrm{h}) ; x$ is the spatial coordinate $(\mathrm{cm})$; and $\omega$ is the mass transfer coefficient $\left(\mathrm{h}^{-1}\right)$. The general boundary conditions are given by:

$C_{i}=0 ; \quad 0<x<l, \quad t=0$

$V_{i} C_{0}=-D_{i} \frac{\partial C_{i}}{\partial x}+V_{i} C_{i}$

$0=-D_{i} \frac{\partial C_{i}}{\partial x}+V_{i} C_{i} ; \quad x=0, \quad t>t_{\mathrm{p}}$

$\partial C_{i} / \partial x=0 ; \quad x=l, \quad t>0$

The initial and boundary conditions of continuous input used in this study can be expressed as:

$C=0 \quad$ or $\quad C_{i}=0 ; \quad 0<x<l, \quad t=0$

$V C_{0}=K \quad$ or $\quad V_{i} C_{0}=-D_{i} \frac{\partial C_{i}}{\partial x}+V_{i} C_{i} ; \quad x=0, \quad t>0$

$\frac{\partial C}{\partial x}=0 \quad$ or $\quad \frac{\partial C_{i}}{\partial x}=0 ; \quad x=l, \quad t>0$

where $D_{i}$ is the dispersion coefficient in the mobile region; $V$ and $V_{i}\left(C\right.$ and $\left.C_{i}\right)$ are the pore water velocities in the soil and in the mobile region; $l$ is the length of the experimental soil column $(\mathrm{cm})$; and $t$ is the input time of the solute $(\mathrm{h})$.

Using the derived values of the dispersion coefficients ( $D$ or $D_{i}$ ) from each of the fitted solute transport models, dispersivity, $\lambda(\mathrm{cm})$, and the Peclet number, $P e$, were calculated and defined using the following equations (Peck and Watson 1979):

$\lambda=D / v$ 
$P e=l v / D=l / \lambda$.

\section{Column experiments}

Four undisturbed soil columns $(29.45 \mathrm{~cm}$ diameter, $45 \mathrm{~cm}$ long) were obtained from the A and B soil horizons (10-60 cm depth) at the Ansai field station, Shaanxi Province, on the Loess Plateau of China. Selected soil properties were as follows: $0.60 \mathrm{~kg} \mathrm{~kg}^{-1}$ silt content; $0.27 \mathrm{~kg} \mathrm{~kg}^{-1}$ clay content; $0.005 \mathrm{~kg} \mathrm{~kg}^{-1}$ organic matter; $8.00 \mathrm{cmol} \mathrm{kg}^{-1}$ cation exchange capacity; and $5.21 \mathrm{cmol} \mathrm{kg}^{-1} \mathrm{Na}$ exchange capacity. The particle size distribution was determined by sieving in combination with the pipette method; organic matter was determined by potassium dichromate titration; the cation exchange capacity was determined by $\mathrm{BaCl}_{2}$ exchange; and the percentage of exchangeable $\mathrm{Na}$ was determined by $\mathrm{NH}_{4}$ OAc exchange.

Solute transport experiments were conducted to study the movement of a chloride through the soil columns. In the laboratory, the bases of the columns were cut to give the desired soil column length $(42 \mathrm{~cm})$. The outer walls were coated with a thin layer of paraffin wax, and then, each column was inserted into a steel cylinder. The upper surface of each soil column represented the actual field surface except that the litter and loose soil were removed in order to obtain a level surface. Chloride transport experiments were carried out using the miscible displacement method (Selim et al. 1987). Each soil column was saturated over a period of more than 7 days by allowing a $0.005 \mathrm{~mol} \mathrm{~L}^{-1} \mathrm{CaCl}_{2}$ solution, which helps to preserve the soil structure when compared to deionized water, to percolate through the soil from the soil surface. Several pore volumes of solution infiltrated the soil during this time. The water fluxes to be investigated $(3.34,4.18,6.69$, $8.35 \mathrm{~cm} \mathrm{~h}^{-1}$ ) were obtained by using a piston pump before the input of $0.15 \mathrm{~mol} \mathrm{~L}^{-1} \mathrm{CaCl}_{2}$ solution pulses. Following the input of the solute, the effluent was collected in 500-mL volumetric flasks and then titrated with silver nitrate solution to determine the concentration of chloride ion $\left(\mathrm{Cl}^{-}\right)$.

Two types of chloride BTCs were generated. The first type, referred to as a small pulse BTC, was generated by introducing a small pulse $(0.1$ pore volumes of $0.15 \mathrm{~mol} \mathrm{~L}^{-1} \mathrm{CaCl}_{2}$ ) into each soil column; this small chloride pulse was recovered in four pore volumes of $0.005 \mathrm{~mol} \mathrm{~L}^{-1} \mathrm{CaCl}_{2}$ solution after the small pulse application. The second type, referred to as a large pulse BTC, was generated in the same way as the first type except that the duration of the input pulse was longer (about 0.5 pore volumes). All the large pulse input experiments were carried out in the same columns as the small pulse input experiments finished, just after ensuring that the water flow was stable and the concentration of chloride in the effluent was close to 0 .

\section{Results and discussion}

\section{Effect of pore water velocity on breakthrough curves for small pulse input applications}

Figure 1 presents the small pulse BTCs with pore water velocities of $3.34,4.18, \quad 6.69, \quad 8.35 \mathrm{~cm} \mathrm{~h}^{-1}$ using $0.15 \mathrm{~mol} \mathrm{~L}^{-1} \mathrm{CaCl}_{2}$ solution as a tracer. All the BTCs for the different pore water velocities were smooth. The chloride concentration first increased and then decreased with increasing pore volumes of effluent. From Fig. 1, it can be seen clearly that pore water velocity had a considerable effect on the BTCs. With increasing pore water velocity, most of the chloride reached the base of the soil column more rapidly. This also resulted in different dilution when $0.005 \mathrm{~mol} \mathrm{~L}^{-1} \mathrm{CaCl}_{2}$ solution was induced. The peak values in the chloride BTCs increased as the pore water velocity increased, which could be confirmed that the faster the chloride moved in the soil column, the faster it will move to the bottom of the soil column and the least it would be diluted.

The BTCs also showed more significant tailing phenomenon, especially for the slower pore water velocities (Fig. 1). Due to the slower movement of some chloride solution through the soil matrix by convection and diffusion, a fraction of the chloride in the pulse flowed relatively rapidly via the macrospores to the base of the soil column, as a result, the remaining chloride migrated through the soil matrix relatively slowly by diffusion, which led to the chloride appeared in the effluent over a longer period of time as demonstrated by the long tailing. The degree of tailing for the slower pore water velocities can thus be considered to result from a lesser degree of bypass flow and a greater degree of flow through the soil matrix (Bouma and Anderson 1977; Sollins and Radulovich 1988) resulting in an overall slower solute flow. With increasing pore water velocity, the chloride moving by diffusion cannot fully exchange by convection while a greater fraction moves through preferential flow so that the tailing becomes longer. This explanation is consistent with our experimental results.

\section{Effect of pore water velocity on breakthrough curves for large pulse input applications}

Figure 2 presents the large pulse BTCs with pore water velocities of $3.34,4.18, \quad 6.69, \quad 8.35 \mathrm{~cm} \mathrm{~h}^{-1}$ using $0.15 \mathrm{~mol} \mathrm{~L}^{-1} \mathrm{CaCl}_{2}$ solution as a tracer. The BTCs all show that the concentration of chloride initially increased 
Fig. 1 Small pulse input, influences of different pore water velocities on BTCs of the experimental result
Fig. 2 Large pulse input, influences of different pore water velocities on solute breakthrough curves

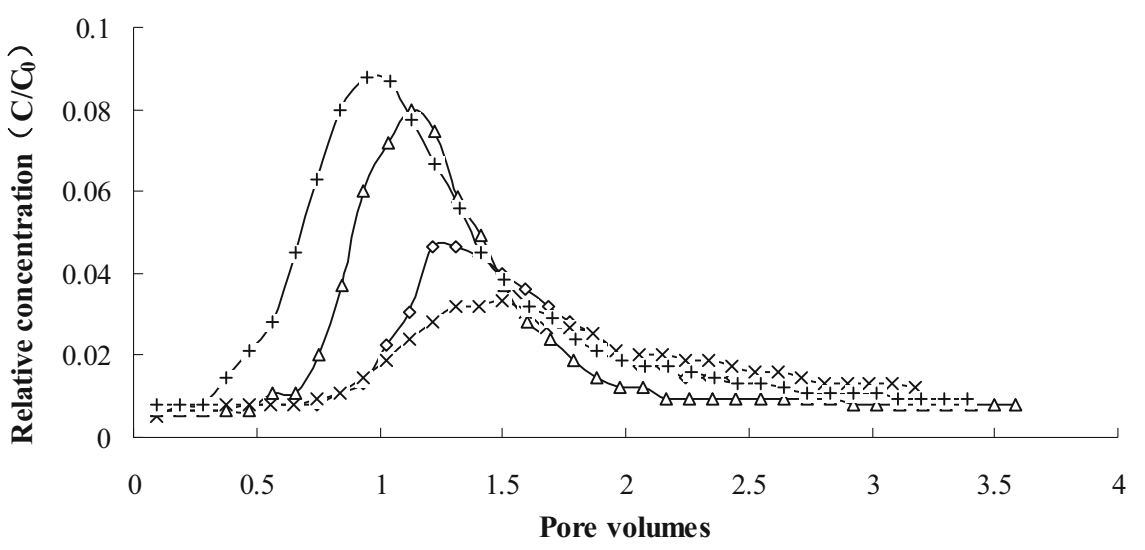

$-\diamond-3.34 \mathrm{~cm} \mathrm{~h}^{-1} \quad-\times-4.18 \mathrm{~cm} \mathrm{~h}^{-1} \quad-\triangle-6.69 \mathrm{~cm} \mathrm{~h}^{-1} \quad-+-8.35 \mathrm{~cm} \mathrm{~h}^{-1}$

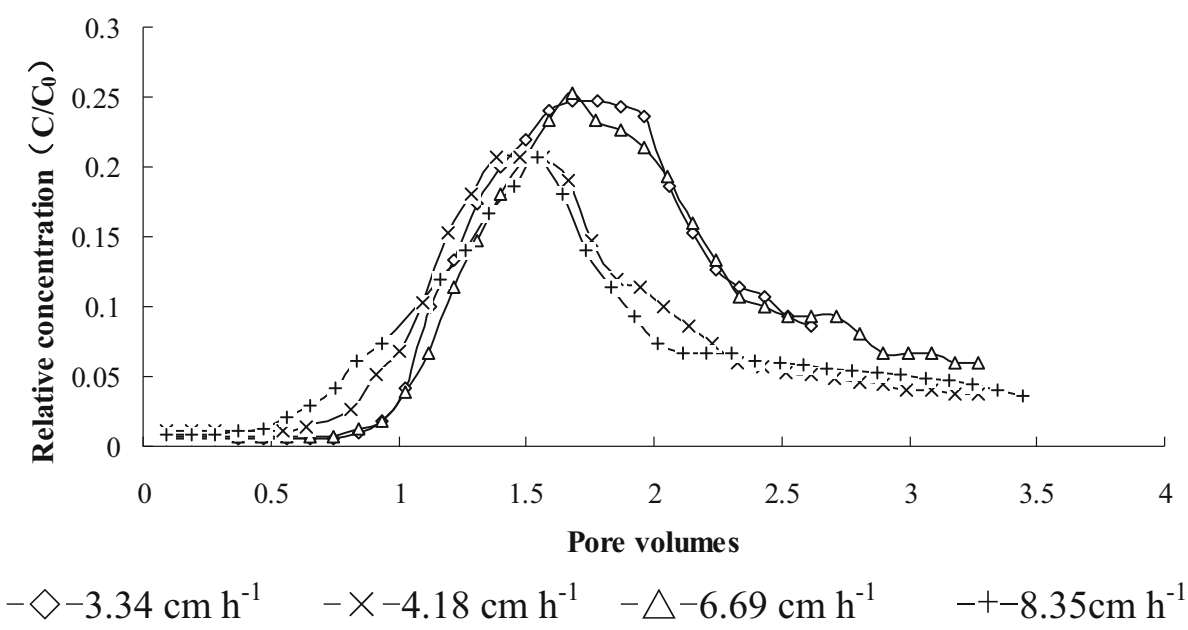

to a peak value shortly after the pulse was applied and then decreased with increasing pore water velocity. All of the BTCs were smooth and had single peaks, which was similar to the small pulse input BTC cases. The maximum concentration values were much higher than those in the small pulse experiment. The more the chloride was induced, the more the concentration could be detected in the effluent.

From Fig. 2, it could also find that the occurrence of asymmetry in the large pulse BTCs was less obvious than that in the small pulse BTCs. This was due to the matrix flow in the miscible displacement process being slow during the pulse, although greater amounts of $\mathrm{Cl}^{-}$entered the matrix, so that it initially had no evident effect on the solute in the effluent. This meant that the primary flow path of the solute initially occurred through the preferential flow path while the secondary flow was through the matrix. Solute entering the soil matrix caused the solute concentration in the matrix to increase gradually. Subsequently, the solute concentration in the effluent via preferential flow rapidly declined while the solute concentration in the matrix decreased relatively slowly. The two differing infiltration processes occurring at different rates were the main causes of the asymmetry observed in the BTCs. However, the greater amounts of solute entering the matrix during the large pulse, compared with the small pulse, resulted in higher concentrations of solute in the later effluent samples due to the secondary pathway and this reduced the asymmetry observed in the BTCs. Notably, there was no secondary pump value observed on the tailing side in our experiments.

\section{Impact of different pulse inputs on, and comparisons between experimental and predicted, breakthrough curves}

In order to further study the influences of different pore water velocities on solute transport, chloride BTCs generated by small and large pulse inputs were fitted by both the CDE and T-R (Fig. 3). All four BTCs showed that the 
Fig. 3 Influences of different input pulse volumes on breakthrough curves and the comparison between experimental data (Exp. Data) and the values fitted by the convection-dispersion equation (CDE) and two-region model
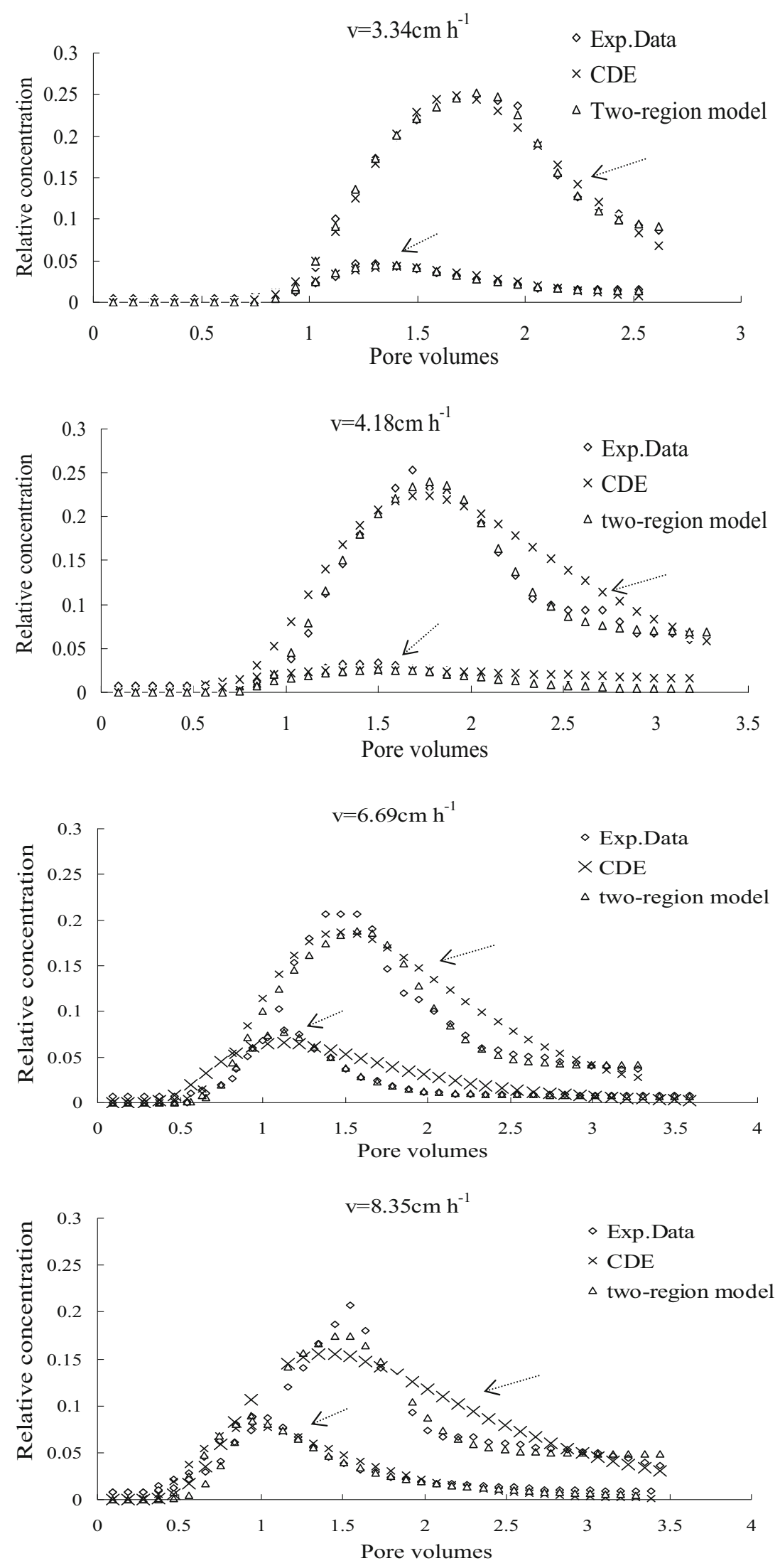
predicted relative concentrations of chloride ions compared favorably with the experimental data (Fig. 3). Therefore, both the CDE and the T-R could well describe all the BTCs.

All the BTCs for both application methods were smooth and had a single peak (Fig. 3). However, the BTCs varied differently, especially with regard to the peak value, according to the application method. The presence of $\mathrm{Cl}^{-}$ in the effluent was detected earlier for the small pulse inputs. It is apparent that the shape of the BTC was dependent on the pulse duration as well as on the pore water velocity. With decreases in pore water velocity, the difference between the peak values of the two application methods became more apparent.

The T-R tended to fit the data better than the CDE; however, the large pulse BTCs were less well fitted than the small pulse BTCs; when the pore velocity increased, the two model could not fit the model well, especially for the tailing part (Fig. 3). Although the two-region domain model could describe the process of solute transport better in undisturbed soil than the CDE, the interpretations of individual peaks were not fully satisfactory. Using the superposition principle, Ma and Selim (1994) found that a small pulse BTC contained as much information on solute transport as a large pulse BTC.

Though the size of the columns is huge enough, the marginal effects on solute transport cannot be completely eliminated and may affect BTCs. Possibly the way in which large and small pores are distributed within the studied loessial soil, as well as the pore water velocity, are important influencing factors that determine whether one or two peaks occur.

\section{Effect of different pulse input methods and pore water velocities on model parameters}

In order to further study the effect of pulse input and pore water velocity on the solute transport process, the fitted parameters of the CDE and of the T-R were examined (Table 1). In the CDE, the retardation factor, $R$, was fitted to the BTCs, while in the T-R, there were two fitted parameters, i.e., the mobile-immobile partition coefficient, $\beta$, and the mass transfer coefficient, $\omega$. From the two fitted models, the Peclet number, $P e$, and the dispersivity, $\lambda(\mathrm{cm})$, were determined from the values obtained for the dispersivity coefficients (Table 1). Since the processes involved with the large pulse input method were slower, the data obtained from these experiments were used to determine the model parameters, and since the input time was shorter for the small pulse input method, the data from these experiments were used to assess the prediction accuracy. Therefore, only the effect of pore water velocity on the parameters for the large pulse input method will be discussed.

From Table 1, the two models' prediction accuracy in describing solute transport can be compared. The SSQ values indicated that both models predicted values for relative concentrations of $\mathrm{Cl}^{-}$that well fitted the experimental data. However, in general, the T-R fits the experimental data slightly better than the CDE. This can be attributed to the greater number of parameters used in the $\mathrm{T}-\mathrm{R}$, which more accurately described the solute transport processes, than those used in the CDE.

\section{Dispersivity, $\lambda$}

From Table 1, it can be seen that the values of the dispersivity, $\lambda$, calculated from the dispersion coefficients given by the CDE were about one order of magnitude larger than those obtained using the dispersion coefficients from the T-R (Table 1 ). The larger $\lambda$ values result from the definition of $D$ used in the CDE, which is a parameter that combines both dispersion and flow heterogeneity characteristics. The larger $\lambda$ values also resulted in the more extensive tailing of the calculated BTCs (Fig. 2).

\section{Peclet number, Pe}

The Peclet number, $P e$, is the ratio of the characteristic times for hydrodynamic dispersion $\left(l^{2} / D\right)$ and convection $\left(l / v_{\mathrm{m}}\right)$ and depends solely on the medium, provided the contribution to mixing by molecular diffusion is significant. Thus, $P e$ provides a measure of the relative

Table 1 Fitting parameters derived by either the convection-dispersion equation CDE or the two-region model (T-R) in undisturbed soil

\begin{tabular}{|c|c|c|c|c|c|c|c|c|c|c|}
\hline \multirow[t]{2}{*}{ Pore water velocity $\left(\mathrm{cm} \mathrm{h}^{-1}\right)$} & \multicolumn{2}{|l|}{ SSQ } & \multicolumn{2}{|l|}{$\lambda$} & \multicolumn{2}{|l|}{$P e$} & \multicolumn{2}{|l|}{$\beta$} & \multicolumn{2}{|l|}{$\omega$} \\
\hline & $\mathrm{CDE}$ & $\mathrm{T}-\mathrm{R}$ & $\mathrm{CDE}$ & $\mathrm{T}-\mathrm{R}$ & $\mathrm{CDE}$ & $\mathrm{T}-\mathrm{R}$ & $\mathrm{CDE}$ & $\mathrm{T}-\mathrm{R}$ & $\mathrm{CDE}$ & $\mathrm{T}-\mathrm{R}$ \\
\hline 3.34 & $2.57 \mathrm{E}-03$ & 7.03E-04 & 0.23 & 0.069 & 185.06 & 607.27 & - & 0.19 & - & 1.66 \\
\hline 4.18 & $2.26 \mathrm{E}-02$ & $2.14 \mathrm{E}-03$ & 0.37 & 0.041 & 114.00 & 1014.79 & - & 0.17 & - & 1.61 \\
\hline 6.69 & $1.87 \mathrm{E}-02$ & $7.51 \mathrm{E}-03$ & 0.65 & 0.046 & 64.59 & 903.47 & - & 0.082 & - & 1.87 \\
\hline 8.35 & $1.69 \mathrm{E}-02$ & $4.38 \mathrm{E}-03$ & 0.74 & 0.16 & 56.75 & 261.72 & - & 0.099 & - & 1.99 \\
\hline
\end{tabular}

SSQ sum of squares residual, $\lambda$ dispersivity $(\mathrm{cm}), P e$ Peclet number, $\beta$ mobile-immobile partition coefficient, $\omega$ mass transfer coefficient 
importance of advective and dispersive processes. The values of $P e$, determined by the dispersion coefficient derived from either the $\mathrm{CDE}$ or the T-R, reflected the inverse relationship between $P e$ and $\lambda$ (Table 1).

Comparison of the $P e$ values in Table 1 shows that the Peclet number calculated with Eq. (17) generally followed the same trend for both models (except for $3.34 \mathrm{~cm} \mathrm{~h}^{-1}$ ), decreasing with increases in velocity, and hence being largest for the $3.34 \mathrm{~cm} \mathrm{~h}^{-1}$ by the CDE as well as for the $4.18 \mathrm{~cm} \mathrm{~h}^{-1}$ by the T-R. In this study, $P e$ estimated from the fitted parameters of both models was also sensitive to different degrees, and the values predicted by the T-R are much larger than those yielded by the $\mathrm{CDE}$, to the various experimental conditions imposed on the same, or on different columns. The Peclet number provides a measure of the movement of the solute by mass flow moving vertically in the columns, while dispersion involves advective movement and the values obtained indicate that the advective process dominates (Schulin and van Genuchten 1987). Thus, in all the columns, advective flow was the dominant component of solute transport, especially for the lower velocities (except for $3.34 \mathrm{~cm} \mathrm{~h}^{-1}$ ). It appears that even larger velocity impeded diffusive flow more considerable (Table 1).

\section{Mobile-immobile partition coefficient, $\beta$}

The mobile-immobile partition coefficient, $\beta$, of the T-R represents the fraction of solute present in the mobile region under equilibrium conditions (Schulin and van Genuchten 1987). In the T-R, solute ions in the mobile region can freely move by dispersion but can only move and exchange by diffusion in the immobile region. The values of the mobile-immobile partition coefficient, $\beta$, became smaller with increases in the pore water velocity (Table 1). This was dependent on the initial tracer input in the presence of macrospores, since a portion of the solute transported by preferential flow moves quickly down the soil column without fully interacting with the soil matrix. Increasing pore water velocity results in greater resistance to preferential flow through the macrospores, so that convection and diffusion of ions in the mobile region will be reduced relative to the immobile region, resulting in reductions in the value of the mobile-immobile partition coefficient, $\beta$.

In many studies, the mobile water fraction has been assumed to be constant (Rao et al. 1980; Bajracharya and Barry 1997). However, experimental data from other studies showed that $\beta$ tended to decrease with increasing pore water velocity when using curve-fitting procedures $(\mathrm{Li}$ et al. 1994; Seyfried and Rao 1987). This is consistent with the experimental results of this study.

\section{Mass transfer coefficient, $\omega$}

The mass transfer coefficient, $\omega$, characterizes the level of solute exchange between mobile and immobile regions. The mass transfer coefficient, $\omega$, increased with increases in pore water velocity (Table 1). DeSmedt (1979) and Wierenga et al. (1996) found a linear relationship between the mass transfer area coefficient, $\omega_{\mathrm{a}}$, and the pore water velocity, $v$, in the mobile region given by: $\omega_{\mathrm{a}}=0.042 v+2.2$, which demonstrated that $\omega$ would also increase as the pore water velocity increased. That finding was consistent with the experimental results of this study. It also demonstrated that $\omega$ was not only dependent on the molecular diffusion coefficient but also on the distance and the cross-sectional area involved during diffusion.

\section{Conclusions}

In the experimental study of an undisturbed loessial soil, notable differences were found for the effect of different input pulses and pore water velocities on the process of solute transport. The effects were also reflected in the model parameters of the CDE and T-R. Both models well fitted the experimental data, although the T-R generally had a higher prediction accuracy. An analysis of the model parameters showed that, when pore water velocity increased, the mobile-immobile partition coefficient tended to decrease and the mass transfer coefficient tended to increase. In order to accurately determine the changes and differences among the various model parameters under different conditions, they should be obtained from replacement experiments, whereby different conditions are applied to the same soil matrix. This form of research should be further advanced to better control conditions.

Acknowledgments The study was financially supported by the National Natural Science Foundation of China (41001132; 51239009), Natural Science Foudation of Shaanxi (2015JQ5161), the Science and Technology Planning Project of Shaanxi Province (2013kjxx-38); the Shaanxi Water Conservancy Project (2013slt-04).

Open Access This article is distributed under the terms of the Creative Commons Attribution 4.0 International License (http:// creativecommons.org/licenses/by/4.0/), which permits unrestricted use, distribution, and reproduction in any medium, provided you give appropriate credit to the original author(s) and the source, provide a link to the Creative Commons license, and indicate if changes were made.

\section{References}

Anderson JL, Bouma J (1977) Water movement through pedal soils: I. Saturated flow. Soil Sci Soc Am J 41:413-418 
Bajracharya K, Barry DA (1997) Non-equilibrium solute transport parameters and their physical significance: numerical and experimental results. J Contam Hydrol 24:185-204

Beven K, Germann P (1982) Macrospores and water flow in soils. Water Resour Res 18:1311-1325

Bouma J, Anderson JL (1977) Water and chloride movement through soil columns simulating pedal soils. Soil Sci Soc Am J 41:766-770

Coats KH, Smith BD (1964) Dead-end pore volume and dispersion in porous media. Soil Sci Soc Pet Eng J 4:73-84

DeSmedt F (1979) Theoretical and experimental study of solute movement through porous media with mobile and immobile water. PhD dissertation, Vrijie Universiteit, Fac Der Toegepaste Wetensch, Dienst Hydrol, Brussels

Elrick DE, French LK (1966) Miscible displacement patterns on disturbed and undisturbed soil cores. Soil Sci Soc Am Proc 30:153-156

Haws N, Das BS (2004) Dual-domain solute transfer and transport processes: evaluation in batch and transport experiments. J Contam Hydrol 75:24

Kanchanasut P, Scotter DR, Tillman RW (1978) Preferential solute movement through larger soil voids. II. Experiments with saturated soil. Aust J Soil Res 16:269-276

Lapidus L, Amundson NR (1952) Mathematics of adsorption in beds, V. Effect of intra-particles diffusion in flow system in fixed beds. J Phys Chem 56:683-6688

Li L, Barry DA, Culligan-Hensleya PJ, Bajracharya K (1994) Mass transfer in soils with local stratification of hydraulic conductivity. Water Resour Res 30:2891-2900

Ma L, Selim HM (1994) Tortuosity, mean residence time and deformation of tritium breakthroughs from soil columns. Soil Sci Soc Am J 58:1076-1085

Peck AJ, Watson JD (1979) Hydraulic conductivity and flow in nonuniform soil. In: Workshop on soil physics and field heterogeneity, CSIRO Div Environ Mech, Canberra
Rao PSC, Rolston DE, Jessupa RE, Davidson JM (1980) Solute transport in aggregated porous media: theoretical and experimental evaluation. Soil Sci Soc Am J 44:1139-1146

Schulin R, van Genuchten MTh (1987) An experimental study of solute transport in a stony field soil. Water Resour Res 23:1785-1794

Selim HM, Schulin R, Fluhler H (1987) Transport and ion exchange of calcium and magnesium in an aggregated soil. Soil Sci Soc Am J 51:876-884

Seyfried MS, Rao PSC (1987) Solute transport in undisturbed columns of an aggregated tropical soil: preferential flow effects. Soil Sci Soc Am J 51:1434-1444

Skopp J, Warrick AW (1974) A two-phase model for the miscible displacement of reactive solutes in soils. Soil Sci Soc Am J 38:545-550

Sollins P, Radulovich R (1988) Effects of soil physical structure on solute transport in a weathered tropical soil. Soil Sci Soc Am J 52:1168-1173

van Genuchten MTh, Wagenet RJ (1989) Two-site/two-region models for pesticide transport and degradation: theoretical development and analytical solutions. Soil Sci Soc Am J 53:1303-1310

van Genuchten MTh, Wierenga PJ (1976) Mass transfer studies in sorbing porous media. I Analytical solution. Soil Sci Soc Am J 40:473-480

White RE (1985) The influence of macrospores on the transport of dissolved and suspended matter through soil. Adv Soil Sci 3:95-120

White RE, Dyson JS, Gerstl Z, Yaron B (1986) Leaching of herbicides through undisturbed cores of a structured clay soil. Soil Sci Soc Am J 50:277-283

Wierenga PJ, Young MH, Mancino CF (1996) Large weighing lysimeters for water use and deep percolation studies. Soil Sci Soc Am J 161:491-501

Zhou BB, Shao MA (2009) Effects of the rock fragments on water movement and solute transport in a Loess Plateau soil. Compt Rendus Geosci 341:462-472 